\title{
Parallel Permittivity Elements for Radio Frequency Waves in Elongated D-shaped Tokamaks
}

\author{
N. I. Grishanov ${ }^{1}$, A. F. D. Loula ${ }^{1}$, C. A. de Azevedo ${ }^{2}$, and J. Pereira Neto ${ }^{2}$ \\ ${ }^{1}$ Laboratório Nacional de Computação Científica, Av. Getúlio Vargas 333, Quitandinha, 25651-075, Petrópolis, RJ, Brazil \\ ${ }^{2}$ Universidade do Estado do Rio de Janeiro, Rua São Francisco Xavier 524, Maracanã, 20550-900, Rio de Janeiro, RJ, Brazil
}

Received on 15 January, 2003; revised version received on 27 April, 2004

\begin{abstract}
Parallel permittivity elements are derived for radio-frequency waves in an axisymmetric tokamak with D-shaped transverse cross-sections of the magnetic surfaces under arbitrary aspect ratio, arbitrary elongation and small triangularity. The bounce resonances are taken into account for untrapped (passing or circulating) and three groups of trapped particles. The corresponding limits for the simpler plasma models are considered. Our dielectric characteristics are suitable to estimate the wave dissipation by electron Landau damping during the plasma heating and current drive generation in the frequency range of Alfvén and fast magnetosonic waves, for both the large and low aspect ratio tokamaks with circular, elliptic and D-shaped magnetic surfaces. The dissipated wave power is expressed by the summation of terms including the imaginary parts of both the diagonal and non-diagonal elements of the parallel permittivity.
\end{abstract}

\section{Introduction}

As is well known, tokamaks represent a promising route to magnetic thermonuclear fusion. In order to achieve the burning conditions in these devices additional plasma heating must be employed. Effective schemes of heating and current drive in magnetized plasmas can be realized using the collisionless dissipation of radio-frequency waves (e.g., Alfvén, fast magnetosonic and lower hybrid waves) by electron Landau damping, transit time magnetic pumping (TTMP), cyclotron and bounce wave-particle interactions etc. Usually, these aspects of the kinetic wave theory are studied by solving the Vlasov-Maxwell's equations. However, for tokamaks, this problem is not simple since to solve the differential wave equations we should use the complicated integral dielectric characteristics valid in the given frequency range for realistic two- or three-dimensional plasma models. The form of the dielectric tensor components, $\epsilon_{i k}$, depends substantially on the geometry of an equilibrium magnetic field and, accordingly, on the chosen geometrical coordinates.

Nonetheless, for the large aspect ratio tokamaks (where the inverse aspect ratio is a small parameter, $\rho / R_{0}<<1$ ), the kinetic wave theory is developed quite fully, see, for example Refs. [1-9] and the bibliography therein. Using the averaged (over magnetic surface $\rho=$ const, i.e., over the poloidal angle $\theta$ ) expressions for $\epsilon_{i k}$ it was possible $[2,3,8,9]$ to perform a general analysis of the dispersion characteristics of the magnetohydrodynamic waves and to estimate the damping rates of the Alfvén and fast magnetosonic waves in various limiting cases. The specific toroidal effects are connected with the fact that in tokamaks (in contrast to a cylindrical plasma confined in the straight or helical magnetic field) the parallel velocity of plasma particles (along the equilibrium magnetic field lines) is not constant, i.e., is modulated depending on $\theta$ for the given $\rho$. Moreover, in their dependence on the pitch angle the plasma particles should be split in the two populations of the so-called trapped (having the stop-points, where their parallel velocity is equal to zero) and the untrapped (or passing, or circulating) particles moving along the equilibrium magnetic field line with a large modulated parallel velocity. Since the trajectories of the trapped and untrapped particles are different, the Vlasov equation should be resolved separately for each particles group. As a result, the trapped and untrapped particles give different contributions to the dielectric tensor elements; the Cherenkov-resonance conditions of an effective interaction of the trapped and untrapped particles are different; and, accordingly, the wave dissipation by the trapped and untrapped particles (mainly, electrons) are different depending of the ratio of the wave phase velocity to the thermal velocity of the untrapped particles/electrons and of the ratio of the wave frequency to the bounce frequency of the trapped particles. One of the interesting features of the toroidal plasmas is the contribution $[5,6]$ of all the spectrum of the electric field to the $m$-th harmonic of the perturbed current density: $4 \pi j_{i}^{m} / \omega=\sum_{m^{\prime}}^{ \pm \infty} \epsilon_{i k}^{m, m^{\prime}} E_{k}^{m^{\prime}}$.

All these features of the large aspect ratio tokamak take place in low aspect ratio toroidal plasmas (see, e.g., Refs. [10-12] for tokamaks with circular transverse cross-sections of the magnetic surfaces). In both the large and low aspect ratio tokamaks with circular magnetic surfaces, the equilibrium magnetic field has only one minimum (or three extremums, with respect to the poloidal angle) in an equatorial plane at the external part of the magnetic surfaces. Accordingly, in such plasma models there is only one group of trapped particles. The main feature of a toroidal plasma with elliptic magnetic surfaces is the fact (see, e.g., Refs. [13-16]) that the equilibrium magnetic field, in the general case, can have two local minimums (or five extremums, with respect to $\theta$ ). As a result, together with the untrapped and 
usual $t$-trapped particles, two additional groups of the socalled $d$-trapped (or double-trapped) particles can appear at such magnetic surfaces if/where the corresponding criterion is satisfied: $\rho / R_{0}<h_{\theta}^{2}\left(b^{2} / a^{2}-1\right)$; here $b / a$ characterizes the elongation, and $h_{\theta}$ is the poloidal component of the unit vector along the equilibrium magnetic field.

Many present-day tokamaks, in particular the spherical ones, have the D-shaped transverse cross-sections of the magnetic surfaces. In this paper, the parallel dielectric permittivity elements are derived for radio-frequency waves in an axisymmetric toroidal collisionless plasma with D-shaped magnetic surfaces under arbitrary aspect ratio, arbitrary elongation, and small triangularity. The drift-kinetic equation is solved separately for untrapped and three groups of trapped particles as a boundary-value problem, using an approach developed for low aspect ratio tokamaks with circular [12], elliptic [16] and D-shaped [17] magnetic surfaces. The limits for the more simple plasma models are considered.

\section{Plasma model and Drift-Kinetic equation}

To describe an axisymmetric D-shaped tokamak we use the quasi-toroidal coordinates $(\rho, \theta, \phi)$ connected with the cylindrical ones $(R, \phi, Z)$ as [17]

$$
\begin{aligned}
R & =R_{0}+\rho \cos \theta-\frac{d \rho^{2}}{a^{2}} \sin ^{2} \theta, \\
\phi & =\phi, \\
Z & =-\frac{b}{a} \rho \sin \theta,
\end{aligned}
$$

where $R_{0}$ is the radius of the magnetic axis; $a$ and $b$ are, respectively, the minor and major semiaxes of the cross-section of the external magnetic surface. In the $(\rho, \theta)$-coordinates, the initial D-shaped cross-sections are transformed to the circles with the corresponding radius $\rho$ in $0 \leq \rho \leq a$; and the cylindrical components of an equilibrium magnetic field $\mathbf{H}$ are

$$
\begin{aligned}
H_{\phi} & =H_{\phi 0} \frac{R_{0}}{R} \\
H_{R} & =H_{\theta 0} \frac{R_{0}}{R} \sin \theta\left(1+\frac{d \rho^{2}}{a^{2}} \cos \theta\right), \\
H_{Z} & =H_{\theta 0} \frac{b R_{0}}{a R} \cos \theta .
\end{aligned}
$$

Here $H_{\phi 0}$ and $H_{\theta 0}$ are, respectively, the toroidal and poloidal magnetic field amplitudes at the given (by $\rho$ ) magnetic surface. Thus, the module $H=|\mathbf{H}|$ of an equilibrium magnetic field is

$$
H(r, \theta)=\frac{\sqrt{H_{\phi 0}^{2}+H_{\theta 0}^{2}}}{g(\rho, \theta)}
$$

where

$$
\begin{aligned}
g(\rho, \theta) & =\frac{1+\epsilon \cos \theta-\delta \epsilon \sin ^{2} \theta}{\sqrt{1+\lambda \cos ^{2} \theta+4 \delta h_{\theta}^{2} \cos \theta \sin ^{2} \theta}} \\
\epsilon & =\frac{\rho}{R} \\
\delta & =\frac{d \rho}{a^{2}} \\
\lambda & =h_{\theta}^{2}\left(\frac{b^{2}}{a^{2}}-1\right) \\
h_{\theta} & =\frac{H_{\theta 0}}{\sqrt{H_{\phi 0}^{2}+H_{\theta 0}^{2}}}
\end{aligned}
$$

In this model, all magnetic surfaces are similar to each other with the same elongation equal to $b / a$; the triangularity is small $d / a<<1$.

To solve the linearized drift-kinetic equation for the perturbed distribution functions,

$$
f(t, \mathbf{r}, \mathbf{v})=f\left(\rho, \theta, v_{\|}, v_{\perp}\right) \exp (-i \omega t+i n \phi)
$$

we use the standard method of switching to new variables associated with conservation integrals of energy, $v_{\|}^{2}+v_{\perp}^{2}=$ const, and magnetic moment, $v_{\perp}^{2} / 2 H=$ const . Introducing the variables $v$ (particle energy) and $\mu$ (squared pitch angle or non-dimensional magnetic moment) in velocity space instead of the parallel, $v_{\|}$, and perpendicular, $v_{\perp}$ , components of the particle velocity:

$$
v^{2}=v_{\|}^{2}+v_{\perp}^{2}, \quad \mu=\frac{v_{\perp}^{2}}{v_{\|}^{2}+v_{\perp}^{2}} g(\rho, \theta),
$$

the drift-kinetic equation for harmonics $f_{s}$,

$$
f\left(\rho, \theta, v_{\|}, v_{\perp}\right)=\sum_{s= \pm 1} f_{s}(\rho, \theta, v, \mu),
$$

in the zeroth order over the magnetization parameter, after averaging over the gyrophase angle in velocity space, can be reduced to the first order differential equation with respect to poloidal angle $\theta$ :

$$
\begin{gathered}
\sqrt{\frac{1-\mu / g(\rho, \theta)}{1+\lambda \cos ^{2} \theta+4 \delta h_{\theta}^{2} \cos \theta \sin ^{2} \theta}}\left(\frac{\partial f_{s}}{\partial \theta}+\frac{i n \epsilon h_{\phi} f_{s} / h_{\theta}}{1+\epsilon \cos \theta-\delta \epsilon \sin ^{2} \theta}\right) \\
\quad-i s \frac{\rho \omega}{v h_{\theta}} f_{s}=\frac{e \rho E_{\|} F}{T h_{\theta}} \sqrt{1-\frac{\mu}{g(\rho, \theta)}}
\end{gathered}
$$


where

$$
F=\frac{N}{\pi^{1.5} v_{T}^{3}} \exp \left(-\frac{v^{2}}{v_{T}^{2}}\right), \quad v_{T}=\sqrt{\frac{2 T}{M}},
$$

$E_{\|}=\mathbf{E} \cdot \mathbf{h}$ is the parallel to $\mathbf{h}=\mathbf{H} / H$ perturbed electric field component; the steady-state distribution function $F$ is given as a Maxwellian with the particle density $N$, temperature $T$, charge $e$ and mass $M$. The index of particle species (ions and electrons) is omitted in Eq. (9). By $s= \pm 1$ we distin- guish the perturbed distribution functions, $f_{s}$, with positive and negative values of the parallel (relative to $\mathbf{H}$ ) velocity,

$$
v_{\|}=s v \sqrt{1-\frac{\mu}{g(\rho, \theta)}} .
$$

After solving Eq. (9), the contribution of the unspecified kind of particles to the parallel (to $\mathbf{H}$ ) component of the perturbed current density, $j_{\|}=\mathbf{j} \cdot \mathbf{h}$, can be expressed as

$$
j_{\|}(\rho, \theta)=\frac{\pi e}{g(\rho, \theta)} \sum_{s= \pm 1} s \int_{0}^{\infty} v^{3} \int_{0}^{g(\rho, \theta)} f_{s}(\rho, \theta, v, \mu) d \mu d v
$$

where the perturbed distribution function is integrated over all the phase volume of plasma particles in velocity space.

In this paper we solve Eq. (9) in the case when untrapped and three groups of trapped particles can exist in the D-shaped elongated tokamak. In this case, analyzing the conditions $v_{\|}(\mu, \theta)=0$, the phase volume of plasma particles should be split in the phase volumes of untrapped, $t$-trapped and two groups of the $d$-trapped particles by the following inequalities:

$$
\begin{array}{ccc}
0 \leq \mu \leq \mu_{u} & -\pi \leq \theta \leq \pi & - \text { for untrapped particles } \\
\mu_{u} \leq \mu \leq \mu_{t} & -\theta_{t} \leq \theta \leq \theta_{t} & - \text { for } t \text {-trapped particles } \\
\mu_{t} \leq \mu \leq \mu_{d} & -\theta_{t} \leq \theta \leq-\theta_{d} & - \text { for } d \text {-trapped particles } \\
\mu_{t} \leq \mu \leq \mu_{d} & \theta_{d} \leq \theta \leq \theta_{t} & - \text { for } d \text {-trapped particles }
\end{array}
$$

where the reflection points and for $t$ - and $d$-trapped particles can be defined by solving the equation $g(\rho, \theta)=\mu$. The parameters $\mu_{u}, \mu_{t}$, and $\mu_{d}$ are defined as

$$
\begin{aligned}
\mu_{u} & =\mu(\rho, \pm \pi)=\frac{1-\epsilon}{\sqrt{1+\lambda}} \\
\mu_{t} & =\mu(\rho, 0)=\frac{1+\epsilon}{\sqrt{1+\lambda}} \\
\mu_{d} & =\mu\left(\rho, \pm \theta_{\text {min }}\right)
\end{aligned}
$$

where $\pm \theta_{\min }$ are the poloidal angles where the equilibrium magnetic field has two possible minimums satisfying the equation $d g(\rho, \theta) / d \theta=0$. It should be noted, that recently the more general case, when the additional so-called $g$ trapped particles can exist in the D-shaped tokamak, was considered in Ref. [17]. However, under the usual tokamak conditions (small triangularity $d<<a$ and moderate elongation $b / a<2.5)$ these particles are absent in the plasma volume.

The solution of Eq. (9) should be found by the specific boundary conditions of the untrapped and trapped particles. For untrapped particles, we use the periodicity of $f_{s}$ over $\theta$. Whereas, the boundary condition for the $t$ - and $d$ trapped particles is the continuity of $f_{s}$ at the corresponding stop-points $\pm \theta_{t}$ and $\pm \theta_{d}$. As a result, we seek the perturbed distribution functions of the untrapped, $t$ - and $d$-trapped particles (respectively, $f_{s}^{u}, f_{s}^{t}$ and $f_{s}^{d}$ ) as

$$
\begin{aligned}
f_{s}^{u} & =\sum_{p}^{ \pm \infty} f_{s, p}^{u} \exp \left[i 2 \pi(p+n q) \frac{\tau(\theta)}{T_{u}}-i n q \bar{\theta}(\theta)\right], \\
f_{s}^{t}= & \sum_{p}^{ \pm \infty} f_{s, p}^{t} \exp \left[i 2 \pi p \frac{\tau(\theta)}{T_{t}}-i n q \bar{\theta}(\theta)\right], \\
f_{s}^{d} & =\sum_{p}^{ \pm \infty} f_{s, p}^{d} \exp \left[i 2 \pi p \frac{\tau(\theta)-\tau\left(\theta_{d}\right)}{T_{d}}-i n q \bar{\theta}(\theta)\right],
\end{aligned}
$$

where $p$ is the number of the bounce resonances;

$$
\tau(\theta)=\frac{\epsilon+\delta\left(1-\sqrt{1-\epsilon^{2}}\right)}{\epsilon \sqrt{1-\epsilon^{2}}} \int_{0}^{\theta} \frac{1+\epsilon \cos \eta-\epsilon \delta \sin ^{2} \eta}{g(\rho, \eta) \sqrt{1-\mu / g(\rho, \eta)}} d \eta
$$

is the new time-like variable (instead of the poloidal angle $\theta)$ to describe the bounce-periodic motion of untrapped, $t$ and $d$-trapped particles along the magnetic field line with the corresponding bounce periods proportional, respectively, to

$$
\begin{aligned}
T_{u}=2 \tau(\pi), T_{t}=4 \tau\left(\theta_{t}\right), T_{u}=2\left(\tau\left(\theta_{t}\right)-\tau\left(\theta_{d}\right)\right) & \\
\bar{\theta}(\theta) & =\frac{2(\epsilon+\delta)}{\epsilon+\delta\left(1-\sqrt{1-\epsilon^{2}}\right)} \operatorname{arctg}\left(\sqrt{\frac{1-\epsilon}{1+\epsilon}} \operatorname{tg} \frac{\theta}{2}\right)- \\
& -\frac{\delta \sqrt{1-\epsilon^{2}}}{\epsilon+\delta\left(1-\sqrt{1-\epsilon^{2}}\right)}\left(\theta-\frac{\epsilon \sin \theta}{1+\epsilon \cos \theta}\right)
\end{aligned}
$$


is the new poloidal angle where the magnetic field lines are straight; and

$$
q=\frac{\epsilon h_{\phi}}{h_{\theta} \sqrt{1-\epsilon^{2}}}\left(1+\frac{\delta}{\epsilon}\left(1-\sqrt{1-\epsilon^{2}}\right)\right)
$$

is the tokamak safety factor. The Fourier harmonics $f_{s, p}^{u}$, $f_{s, p}^{t}$ and $f_{s, p}^{d}$ for untrapped, $t$ - and $d$-trapped particles can be readily derived after the corresponding bounce-averaging.

\section{Collisionless wave dissipation}

To evaluate the dielectric tensor elements we use the Fourier expansions of the perturbed current density and electric field over the poloidal angle $\bar{\theta}$ :

$$
\begin{gathered}
j_{\|}(\theta) g(\rho, \theta)=\sum_{m}^{ \pm \infty} j_{\|}^{m}(\rho) \exp [i m \bar{\theta}(\theta)], \\
E_{\|}(\theta) \frac{\left(1+\epsilon \cos \theta-\epsilon \delta \sin ^{2} \theta\right)^{2}}{g(\rho, \theta)}
\end{gathered}
$$

$$
=\sum_{m^{\prime}}^{ \pm \infty} E_{\|}^{m^{\prime}}(\rho) \exp \left[i m^{\prime} \bar{\theta}(\theta)\right]
$$

As a result, the whole spectrum of electric field, $E_{\|}^{m^{\prime}}$ by $\sum_{m^{\prime}}$, is present in the given $m$-th harmonic $j_{\|}^{m}$ of the current density:

$$
\frac{4 \pi i}{\omega} j_{\|}^{m}=\sum_{m^{\prime}}^{ \pm \infty} \epsilon_{\|}^{m, m^{\prime}} E_{\|}^{m^{\prime}}
$$

$$
=\sum_{m^{\prime}}^{ \pm \infty}\left(\epsilon_{\|, u}^{m, m^{\prime}}+\epsilon_{\|, t}^{m, m^{\prime}}+\epsilon_{\|, d}^{m, m^{\prime}}\right) E_{\|}^{m^{\prime}}
$$

where $\epsilon_{\|, u}^{m, m^{\prime}}, \epsilon_{\|, t}^{m, m^{\prime}}$ and $\epsilon_{\|, d}^{m, m^{\prime}}$ are the separate contributions of untrapped, $t$-trapped and $d$-trapped particles to the longitudinal (parallel) dielectric permittivity elements, respectively. Here we have taken into account that the two groups of the $d$-trapped particles are symmetric in the phase volume and give the same contribution to $\epsilon_{\|}^{m, m^{\prime}}$. As a result, the elements $\epsilon_{\|, u}^{m, m^{\prime}}, \epsilon_{\|, t}^{m, m^{\prime}}, \epsilon_{\|, d}^{m, m^{\prime}}$ can be expressed as

$$
\begin{aligned}
\epsilon_{\|, u}^{m, m^{\prime}} & =\frac{\omega_{L}^{2} \rho^{2}}{2 \pi^{3} h_{\theta}^{2} v_{T}^{2}} \sum_{p}^{ \pm \infty} \int_{0}^{\mu_{u}} \frac{T_{u} A_{p}^{m} A_{p}^{m^{\prime}}}{(p+n q)^{2}}\left[1+2 u_{p}^{2}+2 i \sqrt{\pi} u_{p}^{3} W\left(u_{p}\right)\right] d \mu, \\
\epsilon_{\|, t}^{m, m^{\prime}} & =\frac{\omega_{L}^{2} \rho^{2}}{2 \pi^{3} h_{\theta}^{2} v_{T}^{2}} \sum_{p}^{ \pm \infty} \int_{\mu_{u}}^{\mu_{t}} \frac{T_{t}}{p^{2}} B_{p}^{m} B_{p}^{m^{\prime}}\left[1+2 v_{p}^{2}+2 i \sqrt{\pi} v_{p}^{3} W\left(v_{p}\right)\right] d \mu, \\
\epsilon_{\|, d}^{m, m^{\prime}} & =\frac{\omega_{L}^{2} \rho^{2}}{\pi^{3} h_{\theta}^{2} v_{T}^{2}} \sum_{p}^{ \pm \infty} \int_{\mu_{t}}^{\mu_{d}} \frac{T_{d}}{p^{2}}\left(C_{p}^{m} C_{p}^{m^{\prime}}+D_{p}^{m} D_{p}^{m^{\prime}}\right)\left[1+2 z_{p}^{2}+2 i \sqrt{\pi} z_{p}^{3} W\left(z_{p}\right)\right] d \mu,
\end{aligned}
$$

where the following definitions are used:

$$
\begin{gathered}
\omega_{L}^{2}=\frac{4 \pi N e^{2}}{M}, \quad u_{p}=\frac{\omega \rho \sqrt{1-\epsilon^{2}} \tau(\pi)}{h_{\theta}|p+n q| v_{T} \pi}\left(1-\frac{\delta}{\epsilon}\left(1-\sqrt{1-\epsilon^{2}}\right)\right) \\
v_{p}=\frac{2 \omega \rho \sqrt{1-\epsilon^{2}} \tau\left(\theta_{t}\right)}{h_{\theta} p v_{T} \pi\left(1+\frac{\delta}{\epsilon}\left(1-\sqrt{1-\epsilon^{2}}\right)\right)}, \quad z_{p}=\frac{\omega \rho \sqrt{1-\epsilon^{2}}\left[\tau\left(\theta_{t}\right)-\tau\left(\theta_{d}\right)\right]}{h_{\theta} p v_{T} \pi\left(1+\frac{\delta}{\epsilon}\left(1-\sqrt{1-\epsilon^{2}}\right)\right)}, \\
A_{p}^{m}=\frac{\epsilon \sqrt{1-\epsilon^{2}}}{\epsilon+\delta\left(1-\sqrt{1-\epsilon^{2}}\right)} \int_{0}^{\pi} \frac{\cos \left[(m+n q) \bar{\theta}(\eta)-(p+m q) \pi \frac{\tau(\eta)}{\tau(\pi)}\right]}{1+\epsilon \cos \eta-\epsilon \delta \sin ^{2} \eta} d \eta \\
\hat{B}_{p}^{m}=\frac{\epsilon \sqrt{1-\epsilon^{2}}}{\epsilon+\delta\left(1-\sqrt{1-\epsilon^{2}}\right)} \int_{0}^{\theta_{t}} \frac{\cos \left[(m+n q) \bar{\theta}(\eta)-p \frac{\pi \tau(\eta)}{2 \tau\left(\theta_{t}\right)}\right]}{1+\epsilon \cos \eta-\epsilon \delta \sin ^{2} \eta} d \eta \\
B_{p}^{m}=\hat{B}_{p}^{m}+(-1)^{p-1} \hat{B}_{-p}^{m}, \\
C_{p}^{m}=\frac{\epsilon \sqrt{1-\epsilon^{2}}}{\epsilon+\delta\left(1-\sqrt{1-\epsilon^{2}}\right)} \int_{\theta_{d}}^{\theta_{t}} \frac{\sin [(m+n q) \bar{\theta}(\eta)] \cos \left[p \pi \frac{\tau(\eta)-\tau\left(\theta_{d}\right)}{\tau\left(\theta_{t}\right)-\tau\left(\theta_{d}\right)}\right]}{1+\epsilon \cos \eta-\epsilon \delta \sin ^{2} \eta} d \eta \\
D_{p}^{m}=\frac{\epsilon \sqrt{1-\epsilon^{2}}}{\epsilon+\delta\left(1-\sqrt{1-\epsilon^{2}}\right)} \int_{\theta_{d}}^{\theta_{t}} \frac{\sin [(m+n q) \bar{\theta}(\eta)] \sin \left[p \pi \frac{\tau(\eta)-\tau\left(\theta_{d}\right)}{\tau\left(\theta_{t}\right)-\tau\left(\theta_{d}\right)}\right]}{1+\epsilon \cos \eta-\epsilon \delta \sin ^{2} \eta} d \eta
\end{gathered}
$$


Note that the Eqs. (28-30) describe the contribution of any kind of untrapped and trapped particles to the dielectric tensor elements. The corresponding expressions for plasma electrons and ions can be obtained from (28-30) replacing $T, N, M, e$ by the electron $T_{e}, N_{e}, m_{e}, e_{e}$ and ion $T_{i}, N_{i}, M_{i}, e_{i}$ parameters, respectively. To obtain the total expressions of the permittivity elements, as usual, it is necessary to sum over all species of plasma particles. The same comments should be addressed to calculate the contribution of ions and electrons to the total current density components using Eq. (12).

As an important feature of the permittivity elements, Eqs. (28-30), is the fact that, since the phase coefficients $A_{p}^{m}, B_{p}^{m}, C_{p}^{m}$ and $D_{p}^{m}$ are independent of the wave frequency $\omega$ and the particle energy $v$, the analytical Landau integration of the perturbed distribution functions of both the trapped and untrapped particles in velocity space is possible. As a result, the parallel permittivity elements are written by the summation of bounce-resonant terms including the well known plasma dispersion function $W(z)$, i.e, by the probability integral of complex argument, Eq. (35). After this, the numerical estimations of both the real and imaginary parts of the parallel permittivity elements become simpler, and their dependence on the wave frequency is defined only by the arguments $u_{p}, v_{p}$ and $z_{p}$ of the plasma dispersion functi- ons, $W\left(u_{p}\right), W\left(v_{p}\right)$ and $W\left(z_{p}\right)$. It should be noted that the drift-kinetic equation, Eq. (9), and the dielectric characteristics, Eqs. (28-30), are derived neglecting the drift effects and the finite particle-orbit widths. These effects (as well as the finite pressure and Larmor radius corrections) can be accounted for in the next order(s) of perturbations over the magnetization parameter. The expressions (28-30) have a natural limit to the corresponding results for tokamaks with elliptic magnetic surfaces (section IV) if $d=0$, and circular magnetic surfaces (section $\mathrm{V}$ ) if $b=a$ and $\lambda \rightarrow 0$.

Our dielectric characteristics can be applied for both large and low aspect ratio tokamaks with D-shaped magnetic surfaces to study the wave processes with a regular frequency such as the wave propagation and wave dissipation during the plasma heating and current drive generation; when the wave frequency is specified, e.g., by the antennagenerator parameters. As was mentioned above, one of the main mechanisms of the radio-frequency plasma heating is the electron Landau damping of radio-frequency waves due to the Cherenkov-resonance interaction of $E_{\|}$with the trapped and untrapped electrons. As a result, after averaging in time and poloidal angle, the wave power absorbed due to the trapped and untrapped electrons, $P=\operatorname{Re}\left(E_{\|} \cdot j_{\|}^{*}\right)$, can be estimated by the expression

$$
P_{\|}=\frac{\omega}{8 \pi} \sum_{m}^{ \pm \infty} \sum_{m^{\prime}}^{ \pm \infty}\left(\operatorname{Im} \epsilon_{\|, u}^{m, m^{\prime}}+\operatorname{Im} \epsilon_{\|, t}^{m, m^{\prime}}+\operatorname{Im} \epsilon_{\|, d}^{m, m^{\prime}}\right)\left[\operatorname{Re} E_{\|}^{m} \operatorname{Re} E_{\|}^{m^{\prime}}+\operatorname{Im} E_{\|}^{m} \operatorname{Im} E_{\|}^{m^{\prime}}\right]
$$

where $\operatorname{Im} \epsilon_{\|, u}^{m, m^{\prime}}, \operatorname{Im} \epsilon_{\|, t}^{m, m^{\prime}}$, and $\operatorname{Im} \epsilon_{\|, d}^{m, m^{\prime}}$ are, respectively, the contributions of untrapped $(u), t$ - and $d$-trapped electrons to the imaginary part of the parallel permittivity elements:

$$
\begin{aligned}
\operatorname{Im} \epsilon_{\|, u}^{m, m^{\prime}} & =\frac{\omega_{L}^{2} \rho^{2}}{\pi^{2.5} h_{\theta}^{2} v_{T}^{2}} \sum_{p}^{ \pm \infty} \int_{0}^{\mu_{u}} \frac{T_{u} A_{p}^{m} A_{p}^{m^{\prime}}}{(p+n q)^{2}} u_{p}^{3} \exp \left(-u_{p}^{2}\right) d \mu, \\
\operatorname{Im} \epsilon_{\|, t}^{m, m^{\prime}} & =\frac{\omega_{L}^{2} \rho^{2}}{\pi^{2.5} h_{\theta}^{2} v_{T}^{2}} \sum_{p}^{ \pm \infty} \int_{\mu_{u}}^{\mu_{t}} \frac{T_{t}}{p^{2}} B_{p}^{m} B_{p}^{m^{\prime}} v_{p}^{3} \exp \left(-v_{p}^{2}\right) d \mu, \\
\operatorname{Im} \epsilon_{\|, d}^{m, m^{\prime}} & =\frac{2 \omega_{L}^{2} \rho^{2}}{\pi^{2.5} h_{\theta}^{2} v_{T}^{2}} \sum_{p}^{ \pm \infty} \int_{\mu_{t}}^{\mu_{d}} \frac{T_{d}}{p^{2}}\left(C_{p}^{m} C_{p}^{m^{\prime}}+D_{p}^{m} D_{p}^{m^{\prime}}\right) z_{p}^{3} \exp \left(-z_{p}^{2}\right) d \mu .
\end{aligned}
$$

Thus, we see that for the same wave frequency $\omega$ and the same electric field amplitudes the wave power absorbed, $P=P_{u}+P_{t}+P_{d}$, is defined by the different contributions of the untrapped, $t$-trapped, and $d$-trapped electrons to the imaginary part of the parallel permittivity elements, Eqs. (38-41).

In the simplest case of Toroidicity-induced Alfvén Ei- genmodes [18] (TAEs), describing the coupling of only two harmonics with $m_{o}$ and $m_{o}-1$, the terms with $m_{o}, m_{o}-1$ should be accounted for in Eq. (38) to estimate the TAEs absorption by the trapped and untrapped electrons. As a result, the dissipated power of TAEs by the electron Landau damping is expressed as

$$
P_{\|}=\frac{\omega}{8 \pi} \sum_{m=m_{o}-1}^{m_{o}} \operatorname{Im} \epsilon_{\|}^{m, m}\left|E_{\|}^{m}\right|^{2}+\frac{\omega}{8 \pi} \operatorname{Im} \epsilon_{\|}^{m_{o}, m_{o}-1}\left(\operatorname{Re} E_{\|}^{m_{o}} \operatorname{Re} E_{\|}^{m_{o}-1}+\operatorname{Im} E_{\|}^{m_{o}} \operatorname{Im} E_{\|}^{m_{o}-1}\right)
$$


where $\left|E_{\|}^{m}\right|^{2}=\left(\operatorname{Re} E_{\|}^{m}\right)^{2}+\left(\operatorname{Im} E_{\|}^{m}\right)^{2}$ is the squared module of the $m$-th electric field harmonic. Of course, our dielectric characteristics in Eqs. (28-30) and Eqs. (39-41), can be applied as well to study, e.g., the excitation/dissipation of the Ellipticity-induced Alfvén Eigenmodes [19] (EAEs) in elongated tokamaks when the $\left(m_{o} \pm 2\right)$-harmonics of the electric field and the permittivity elements $\operatorname{Im} \epsilon_{\|, u}^{m_{o}, m_{o} \pm 2}$, $\operatorname{Im} \epsilon_{\|, t}^{m_{o}, m_{o} \pm 2}$, and $\operatorname{Im} \epsilon_{\|, d}^{m_{o}, m_{o} \pm 2}$ should be involved. Note that the non-diagonal elements $\epsilon_{\|}^{m, m^{\prime}}$ are characteristic of a toroidal plasma model. For the one-mode (cylindrical) approximations, when $m=m^{\prime}=m_{o}$, the non-diagonal elements vanish, i.e. $\left.\operatorname{Im} \epsilon_{\|, u}^{m, m^{\prime}}\right|_{m \neq m^{\prime}}=0$, and Eqs. (38) and (42) can be reduced to the well-known expression

$$
P_{\|}=\frac{\omega}{8 \pi} \operatorname{Im} \epsilon_{\|}^{m, m}\left|E_{\|}^{m}\right|^{2} .
$$

On the other hand, the diagonal parallel permittivity element (as well as other dielectric tensor components, respectively) corresponds to the parallel dielectric permittivity averaged over the magnetic surface and can be used to estimate the damping rates of the radio-frequency waves with the help of the dispersion relations (or the eikonal equations) as was made in Refs. [2,3,8] for individual modes $\sim \exp \left[i\left(\int k_{\rho} d \rho+m \theta+n \phi-\omega t\right)\right]$ in a large aspect ratio tokamak with concentric circular magnetic surfaces.

\section{Tokamak with elliptic magnetic surfaces}

In this section, let us consider a tokamak plasma model with elliptic magnetic surfaces. As in a D-shaped tokamak, in the general case, the module of the equilibrium magnetic field, $H(\rho, \theta)$, can have two local minimums with respect to the poloidal angle $\theta$. In this case, the existence criterion of the $d$-trapped particles can be rewritten as

$$
\epsilon<\lambda \quad \text { or } \quad \frac{b}{a}>\sqrt{1+\epsilon+q^{2} \frac{1-\epsilon^{2}}{\epsilon}} .
$$

Otherwise, if $\epsilon>\lambda$, the equilibrium magnetic field has only one minimum and the $d$-trapped particles are absent at the given magnetic surface (as it is in tokamaks with circular magnetic surfaces where $b=a, \lambda=0$ and, accordingly, $\lambda<\epsilon)$.

The separate contribution of untrapped, $t$-trapped and $d$-trapped particles to the parallel permittivity elements $\left(\epsilon_{\|, u}^{m, m^{\prime}}, \epsilon_{\|, t}^{m, m^{\prime}}\right.$ and $\left.\epsilon_{\|, d}^{m, m^{\prime}}\right)$ can be described by Eqs. (2830 ), where using the limit $d \rightarrow 0$, the phase coefficients and the arguments of the plasma dispersion functions should be rewritten as

$$
A_{p}^{m}=\int_{0}^{\pi} \cos \left[(m+n q) \eta-(p+n q) \pi \frac{\tau(\eta)}{\tau(\pi)}\right] d \eta
$$

$$
\begin{gathered}
B_{p}^{m}=\int_{0}^{\theta_{t}} \cos \left[(m+n q) \eta-p \frac{\pi \tau(\eta)}{2 \tau\left(\theta_{t}\right)}\right] d \eta+ \\
+(-1)^{p-1} \int_{0}^{\theta_{t}} \cos \left[(m+n q) \eta+p \frac{\pi \tau(\eta)}{2 \tau\left(\theta_{t}\right)}\right] d \eta \\
C_{p}^{m}=\int_{\theta_{d}}^{\theta_{t}} \sin [(m+n q) \eta] \cos \left[p \pi \frac{\tau(\eta)-\tau\left(\theta_{d}\right)}{\tau\left(\theta_{t}\right)-\tau\left(\theta_{d}\right)}\right] d \eta
\end{gathered}
$$

$$
D_{p}^{m}=\int_{\theta_{d}}^{\theta_{t}} \sin [(m+n q) \eta] \sin \left[p \pi \frac{\tau(\eta)-\tau\left(\theta_{d}\right)}{\tau\left(\theta_{t}\right)-\tau\left(\theta_{d}\right)}\right] d \eta
$$

$$
\begin{gathered}
\tau(\bar{\theta})=\int_{0}^{\bar{\theta}} \frac{\left(1-\epsilon^{2}\right) G(\rho, \eta) d \eta}{(1-\epsilon \cos \eta)^{2} \sqrt{1-\mu G(\rho, \eta)}} \\
G(\rho, \bar{\theta})=\frac{1}{g(\rho, \theta)}=\frac{\sqrt{(1-\epsilon \cos \bar{\theta})^{2}+\lambda(\epsilon-\cos \bar{\theta})^{2}}}{1-\epsilon^{2}}
\end{gathered}
$$

$$
\bar{\theta}(\theta)=2 \operatorname{arctg}\left[\sqrt{\frac{1-\epsilon}{1+\epsilon}} \operatorname{tg}\left(\frac{1}{2} \operatorname{arctg}\left(\frac{a}{b} \operatorname{tg} \theta\right)\right)\right]
$$

$$
\begin{aligned}
& \theta_{t}=\arccos \left\{\frac{\epsilon(1+\lambda)}{\lambda+\epsilon^{2}}-\sqrt{\frac{\epsilon^{2}(1+\lambda)^{2}}{\left(\lambda+\epsilon^{2}\right)^{2}}-\frac{1}{\lambda+\epsilon^{2}}\left[1+\epsilon^{2} \lambda-\left(\frac{1-\epsilon^{2}}{\mu}\right)^{2}\right]}\right\} \\
& \theta_{d}=\arccos \left\{\frac{\epsilon(1+\lambda)}{\lambda+\epsilon^{2}}+\sqrt{\frac{\epsilon^{2}(1+\lambda)^{2}}{\left(\lambda+\epsilon^{2}\right)^{2}}-\frac{1}{\lambda+\epsilon^{2}}\left[1+\epsilon^{2} \lambda-\left(\frac{1-\epsilon^{2}}{\mu}\right)^{2}\right]}\right\}, \\
& T_{u}=2 \tau(\pi), \\
& T_{t}=4 \tau\left(\theta_{t}\right), \quad T_{u}=2\left(\tau\left(\theta_{t}\right)-\tau\left(\theta_{d}\right)\right), \\
& \mu_{t}=\frac{1+\epsilon}{\sqrt{1+\lambda}}, \quad \mu_{d}=\sqrt{1+\frac{\epsilon^{2}}{\lambda}},
\end{aligned}
$$




$$
u_{p}=\frac{\omega \rho \sqrt{1-\epsilon^{2}} T_{u}}{2 \pi h_{\theta}|p+n q| v_{T}}, \quad v_{p}=\frac{\omega \rho \sqrt{1-\epsilon^{2}} T_{t}}{2 \pi h_{\theta} p v_{T}}, \quad z_{p}=\frac{\omega \rho \sqrt{1-\epsilon^{2}} T_{d}}{2 \pi h_{\theta} p v_{T}}
$$

The more detailed evaluation of the $\epsilon_{\|, u}^{m, m^{\prime}}, \epsilon_{\|, t}^{m, m^{\prime}}$ and $\epsilon_{\|, d}^{m, m^{\prime}}$ dielectric tensor elements in a tokamak with elliptic magnetic surfaces has been done in Ref. [16].

\section{Tokamak with circular magnetic surfaces}

Note that Eqs. (28-37) have been written in the quite general form where the ellipticity and triangularity are accounted for implicitly by the functions $\lambda(\rho), g(\rho, \theta)$ and $\tau(\rho, \theta)$. As for tokamaks with circular magnetic surfaces, where $d=0$ and $\lambda=0$, the expressions $\epsilon_{\|, u}^{m, m^{\prime}}$ and $\epsilon_{\|, t}^{m, m^{\prime}}$ (as well as the corresponding phase coefficients $A_{p}^{m}, B_{p}^{m}$, and other defi- nitions) can be simplified substantially because the $\tau(\rho, \theta)$ functions for the trapped and untrapped particles, see Eqs. $(21,49)$, can be reduced to i) the third kind elliptic integrals in low $(\epsilon<1)$ aspect ratio tokamaks [12], or ii) the first kind elliptic integrals in large $(\epsilon<<1)$ aspect ratio tokamaks [9] As was mentioned above, the $d$-trapped particles are absent in a tokamak with circular magnetic surfaces, therefore $\epsilon_{\|, d}^{m, m^{\prime}}=0$. As a result, in tokamaks with circular magnetic surfaces, e.g., such as TCABR (Tokamak Chauffage Alfvén Brésilien) at the São Paulo University, the expressions (24)(26), (34)-(36) can be reduced to the corresponding results in Ref. [12]. In particular, if $b=a$ and $\lambda \rightarrow 0, d \rightarrow 0$, the contribution of untrapped and $t$-trapped particles to the parallel permittivity elements are

$$
\begin{array}{r}
\epsilon_{\|, u}^{m, m^{\prime}}=\frac{2 \omega_{L}^{2} \rho^{2} \sqrt{\kappa_{o}}(1+\epsilon)}{\pi^{3} h_{\theta}^{2} v_{T}^{2}(1-\text { epsilon })} \sum_{p}^{ \pm \infty} \int_{0}^{1} \frac{\Pi\left(\kappa_{o}, \kappa, \pi / 2\right) A_{p}^{m} A_{p}^{m^{\prime}}}{(p+n q)^{2}\left(\kappa_{o}+\kappa\right)^{1.5}}\left[1+2 u_{p}^{2}+2 i \sqrt{\pi} u_{p}^{3} W\left(u_{p}\right)\right] d \kappa, \\
\epsilon_{\|, t}^{m, m^{\prime}}=\frac{4 \omega_{L}^{2} \rho^{2} \sqrt{\kappa_{o}}(1+\epsilon)}{\pi^{3} h_{\theta}^{2} v_{T}^{2}(1-\epsilon)} \sum_{p=1}^{\infty} \int_{0}^{1} \frac{\Pi\left(\kappa_{o} \hat{\kappa}, \hat{\kappa}, \pi / 2\right) B_{p}^{m} B_{p}^{m^{\prime}}}{p^{2}\left(1+\kappa_{o} \hat{\kappa}\right)^{1.5}}\left[1+2 v_{p}^{2}+2 i \sqrt{\pi} v_{p}^{3} W\left(v_{p}\right)\right] d \hat{\kappa},
\end{array}
$$

where

$$
\begin{gathered}
A_{p}^{m}(\kappa)=\int_{0}^{\pi} \cos \left[(m+n q) \eta-\pi(p+n q) \frac{\Pi\left(\kappa_{o}, \kappa, \eta / 2\right)}{\Pi\left(\kappa_{o}, \kappa, \pi / 2\right)}\right] d \eta, \\
B_{p}^{m}(\hat{\kappa})=\int_{0}^{\theta_{t}} \cos \left[(m+n q) \eta-\pi p \frac{\Pi\left(\kappa_{o} \hat{\kappa}, \hat{\kappa}, \arcsin \left(\sqrt{\frac{1}{\hat{\kappa}}} \sin \frac{\eta}{2}\right)\right]}{2 \Pi\left(\kappa_{o} \hat{\kappa}, \hat{\kappa}, \pi / 2\right)}\right] d \eta+ \\
+(-1)^{p-1} \int_{0}^{\theta_{t}} \cos \left[(m+n q) \eta+\pi p \frac{\Pi\left(\kappa_{o} \hat{\kappa}, \hat{\kappa}, \arcsin \left(\sqrt{\frac{1}{\hat{\kappa}}} \sin \frac{\eta}{2}\right)\right.}{2 \Pi\left(\kappa_{o} \hat{\kappa}, \hat{\kappa}, \pi / 2\right)}\right] d \eta, \\
\kappa_{o}=\frac{2 \epsilon}{1-\epsilon}, \quad \begin{array}{c}
\kappa \\
\theta_{t}(\hat{\kappa})=2 \arcsin (\sqrt{\hat{\kappa}}), \quad \frac{1}{(1-\epsilon)(1+\epsilon-\mu)}, \\
u_{p}(\kappa)=\frac{\omega \rho \sqrt{2(1+\epsilon)\left(\kappa_{o}+\kappa\right)}}{|p+n q| \pi h_{\theta} v_{T} \sqrt{\epsilon}} \Pi\left(\kappa_{o}, \kappa, \frac{\pi}{2}\right), \\
v_{p}(\hat{\kappa})=\frac{2 \omega \rho \sqrt{2(1+\epsilon)\left(1+\kappa_{o} \hat{\kappa}\right)}}{p \pi h_{\theta} v_{T} \sqrt{\epsilon}} \Pi\left(\kappa_{o} \hat{\kappa}, \hat{\kappa}, \frac{\pi}{2}\right) .
\end{array},
\end{gathered}
$$

Introduction of the third kind elliptic integrals, $\Pi(\alpha, \beta, \gamma)$, instead of the $\tau(\theta)$ variable and the bounce periods of untrapped and trapped particles, is convenient for computati- ons since the complete and incomplete elliptic integrals can be used as the standard elementary functions in such advanced mathematical programs as Mathematica, Mathcad and 
Maple. In particular, for the untrapped particles as follows from Eqs. $(49,50)$ if $a=b$

$$
\tau(\bar{\theta})=\sqrt{\frac{2\left(\kappa_{o}+\kappa\right)}{\epsilon(1-\epsilon)}} \Pi\left(\kappa_{o}, \kappa, \frac{\bar{\theta}}{2}\right) .
$$

Moreover, the phase coefficients $A_{p}^{m}$ and $B_{p}^{m}$ can also be calculated by using the Jacobi elliptic functions. In particular, after introducing the new variable $w(\kappa, \theta)$ as the first kind incomplete elliptic integral

$$
w(\kappa, \theta)=\int_{0}^{\theta} \frac{d \eta}{\sqrt{1-\kappa \sin ^{2} \eta}},
$$

the so-called $w$-amplitude function is defined as the inverse transformation, i.e., $\theta=\operatorname{am}(\kappa, w)$, and the Jacobi elliptic functions are

$$
\begin{aligned}
& \operatorname{sn}(\kappa, w)=\sin (\theta)=\sin (\operatorname{am}(\kappa, w)), \\
& \operatorname{cn}(\kappa, w)=\cos (\theta)=\cos (\operatorname{am}(\kappa, w)), \\
& \operatorname{dn}(\kappa, w)=\sqrt{1-\kappa \sin ^{2}(\theta)}=\sqrt{1-\kappa \operatorname{sn}^{2}(\kappa, w)} .
\end{aligned}
$$

$$
\begin{aligned}
& A_{p}^{m}(\kappa)=\int_{-K(\kappa)}^{K(\kappa)} \cos \left[(m+n q) \operatorname{am}(\kappa, w)-\pi(p+n q) \frac{\hat{\Pi}\left(\kappa_{o}, \kappa, \eta / 2\right)}{\hat{\Pi}\left(\kappa_{o}, \kappa, \pi / 2\right)}\right] \operatorname{dn}(\kappa, w) d w \\
& B_{p}^{m}(\kappa)=\sqrt{\kappa} \int_{-2 K(\kappa)}^{2 K(\kappa)} \cos \left[(m+n q) \arcsin (\sqrt{\kappa} \operatorname{sn}(\kappa, w))-p \frac{0.5 \pi \Pi\left(\kappa_{o} \kappa, \kappa, w\right)}{\Pi\left(\kappa_{o} \kappa, \kappa, K(\kappa)\right)}\right] \operatorname{cn}(\kappa, w) d w
\end{aligned}
$$

where

$$
\begin{aligned}
K(\kappa) & =\int_{0}^{\pi / 2} \frac{d \eta}{\sqrt{1-\kappa \sin ^{2} \eta}}, \\
\hat{\Pi}\left(\kappa_{o}, \kappa, w\right) & =\int_{0}^{w} \frac{d u}{\left(1+\kappa_{o} \mathrm{sn}^{2}(\kappa, u)\right.}
\end{aligned}
$$

and the new variables $w$ have been introduced instead of the poloidal angle $\bar{\theta}$ as

$$
w(\kappa, \bar{\theta})=\int_{0}^{\bar{\theta} / 2} \frac{d \eta}{\sqrt{1-\kappa \sin ^{2} \eta}}
$$

for the untrapped particles, and

$$
w(\kappa, \bar{\theta})=\int_{0}^{\arcsin (\sqrt{1 / \hat{\kappa}} \sin (\bar{\theta} / 2))} \frac{d \eta}{\sqrt{1-\hat{\kappa} \sin ^{2} \eta}}
$$

for the $t$-trapped particles. It should be noted that the umbral 'hat'-symbol is omitted in Eq. (71) and can be omitted as well in Eqs. $(58,60)$. The imaginary parts of the parallel permittivity elements $\epsilon_{\|, u}^{m, m^{\prime}}$ and $\epsilon_{\|, t}^{m, m^{\prime}}$, Eqs. $(57,58)$, for radio-frequency waves in a toroidal plasma with the TCABR tokamak parameters, was analyzed numerically in Ref. [20].

\section{Conclusion}

The parallel permittivity elements, Eqs. (28-30), have been derived for radio-frequency waves by solving the driftkinetic equation for untrapped, $t$-trapped and $d$-trapped particles in an axisymmetric toroidal plasma with D-shaped magnetic surfaces and arbitrary aspect ratio, arbitrary elongation and small triangularity. These dielectric characteristics are expressed by the summation of the bounceresonant terms, which include the double integration in velocity space, the phase coefficients, the standard elementary and quasi-elliptic functions. It is shown that analytical Landau integration can be carried out by introducing the plasma dispersion function, or the probability integral of complex argument.

The imaginary parts of the parallel permittivity elements, Eqs. (39-41), are important to estimate the wave power absorbed by electron Landau damping (e.g., during the plasma heating and current drive generation) in the frequency range much less than the ion cyclotron frequency. The dissipated wave power is expressed, Eq. (38), by the summation of terms including the separate contributions of untrapped, $t$-trapped and $d$-trapped particles to the imaginary parts of both the diagonal and non-diagonal elements of the dielectric permittivity. The parallel permittivity elements evaluated in the paper are suitable for both the large $(\epsilon<<1)$ and low $(\epsilon<1)$ aspect ratio D-shaped tokamaks and valid in a wide range of wave frequencies, mode numbers, and plasma parameters. The expressions (28-30) and (39-41) have a natural limit to the corresponding results for toroidal plasmas with elliptic magnetic surfaces if $d=0$, and with circular magnetic surfaces if $b=a$ or $\lambda \rightarrow 0$. Since the drift-kinetic equation is solved as a boundaryvalue problem, the parallel permittivity elements (28-30, 57, 58) can be applied to study the wave processes with a regular frequency such as the wave propagation and wave dissipation during the plasma heating and current drive genera- 
tion, when the wave frequency is specified by the antennagenerator system. Of course, the best application of our dielectric characteristics is to develop a numerical code to solve the two-dimensional Maxwell's equations in elongated Dshaped tokamaks for electromagnetic fields in the frequency range of Alfvén and fast magnetosonic waves. On the other hand, they can be analyzed independently of the solution of Maxwell's equations.

Note that in analyzing the collisionless wave dissipation by plasma electrons in D-shaped tokamaks one should also take into account other kinetic mechanisms of the waveparticle interactions, such as the TTMP (Transit Time Magnetic Pumping) and/or the cyclotron resonance damping. The corresponding information about these Cherenkovresonance wave-particle interactions is included in the transverse and cross-off dielectric permittivity elements. Thus, to describe these effects in our two-dimensional plasma models all the nine dielectric tensor components should be derived accounting for the finite beta and finite Larmor radius corrections. However, this is a topic of additional investigation.

\section{Acknowledgments}

This research was supported by CNPq of Brazil (Conselho Nacional de Desenvolvimento Científico e Tecnológico, project 300637/01-2).

\section{References}

[1] T. Stix, Nucl. Fusion, 15, 737 (1975).

[2] V. N. Belikov, Ya. I. Kolesnichenko, A.B. Mikhailovskii, and V.A. Yavorskii, Sov. J. Plasma Phys. 3, 146 (1977).

[3] T. D Kaladze, A. I. Pyatak, and K. N. Stepanov, Sov. J. Plasma Phys. 8, 467 (1982).
[4] M. Brambilla and T. Krucken, Nucl. Fusion, 28, 1813 (1988).

[5] A. G. Elfimov and S. Puri, Nucl. Fusion, 30, 1215 (1990).

[6] F. M. Nekrasov, Sov. J. Plasma Phys., 18, 520 (1992).

[7] F. Porcelli, R. Stancievicz, W. Kerner, and H. L. Berk, Phys. Plasmas, 1, 470 (1994).

[8] S. V. Kasilov, A. I. Pyatak, and K. N. Stepanov, Plasma Phys. Reports, 24, 465 (1998).

[9] B. N. Kuvshinov and A. B. Mikhailovskii, Plasma Phys. Reports, 24, 623 (1998).

[10] N. I. Grishanov and F. M. Nekrasov, Sov. J. Plasma Phys. 16, 129 (1990).

[11] F. M. Nekrasov, A. G. Elfimov, C. A. de Azevedo, and A. S. de Assis, Plasma Phys. Control. Fusion, 43, 727 (2001).

[12] N. I. Grishanov, C. A. de Azevedo, and J. P. Neto, Plasma Phys. Control. Fusion, 43, 1003 (2001).

[13] F. M. Nekrasov, A. G. Elfimov, V. S. Tsypin, C. A. de Azevedo, and A. S. de Assis, Czech. J. Phys., 46, 565 (1996).

[14] V. S. Tsypin, A. G. Elfimov, F. M. Nekrasov, C. A. de Azevedo, and A. S. de Assis, Phys. Plasmas, 4, 3635 (1997).

[15] N. I. Grishanov, C. A. de Azevedo, and A. S. de Assis, Plasma Phys. Control. Fusion, 41, 645 (1999).

[16] N. I. Grishanov, G. O. Ludwig, C. A. de Azevedo, and J. P. Neto, Phys. Plasmas, 9, 4089 (2002).

[17] N. I. Grishanov, A. F. D. Loula, C. A. de Azevedo, and J. Pereira Neto, Plasma Phys. Control. Fusion, 44, 645 (2003).

[18] C. Z. Cheng and M. S. Chance, Phys. Fluids, 29, 3695 (1986).

[19] R. Betti and J. P. Freidberg, Phys. Fluids, B3, 1865 (1991).

[20] N. . Grishanov, C. A. de Azevedo, and A. S. de Assis, Braz. J. Phys. 32, 179 (2002). 